\title{
Papéis femininos na propaganda e seus efeitos sobre consumidores brasileiros
}

Female roles in advertising and their effects on Brazilian consumers

Papeles femeninos en la propaganda y sus efectos sobre consumidores brasileños

DOI: https://doi.org/10.1590/1809-58442021306

Fernanda Almeida Marcon ${ }^{1}$

https://orcid.org/0000-0002-6257-1197

Rudimar Antunes da Rocha ${ }^{1}$

https://orcid.org/0000-0003-2554-2896

Ana Maria Simões Ribeiro ${ }^{1}$

http://orcid.org/0000-0001-5807-4510

${ }^{1}$ (Universidade Federal de Santa Catarina, Centro Socioeconômico, Programa de Pós-Graduação em Administração. Florianópolis - SC, Brasil).

\section{Resumo}

Esta pesquisa objetivou analisar as atitudes de adultos brasileiros acerca do retrato de estereótipos femininos na propaganda e seus efeitos na imagem dos anunciantes e na intenção de compra. Para isso, foi aplicado um survey entre novembro e dezembro de 2017, em uma amostra de 519 respondentes. Os dados coletados foram analisados por modelagem de equações estruturais. Concluiu-se que a ofensividade causada por papéis femininos estereotipados na propaganda influenciou a intenção de boicotar os produtos. Confirmou-se que as mulheres apresentam atitudes mais negativas do que os homens quanto aos estereótipos retratados, em termos de ofensividade. Contudo, não houve diferença significativa entre o gênero feminino e masculino quanto ao impacto da ofensividade sobre a imagem da empresa ou a intenção de boicote.

Palavras-chave: Propaganda. Papéis Femininos Estereotipados. Imagem da Empresa. Intenção de Compra. Contexto Brasileiro.

\section{Abstract}

This research aimed to analyze the attitudes of Brazilian adults about the portrayal of female stereotypes in advertising and their effects on advertisers' image and intent to buy. A survey was applied between November and December of 2017 in a sample of 519 respondents. The collected data were analyzed by structural equations modeling. It was concluded that the offensiveness caused 
by stereotyped female roles in advertising influenced the intention to boycott the products. It was confirmed that women present more negative attitudes than men about the stereotypes portrayed, in terms of offensiveness. However, there was no significant difference between male and female regarding the impact of offensiveness on the company image or the intention to boycott.

Keywords: Advertising. Stereotyped Female Roles. Company's Image. Purchase Intention. Brazilian Context.

\section{Resumen}

Esta investigación objetivó analizar las actitudes de adultos brasileños acerca del retrato de estereotipos femeninos en la propaganda y sus efectos en la imagen de los anunciantes y en la intención de compra. Para esto, se aplicó una survey entre noviembre y diciembre de 2017 en una muestra de 519 personas. Los datos recolectados fueron analizados por un modelo de ecuaciones estructurales. Se concluyó que la ofensividad causada por papeles femeninos estereotipados en la propaganda influenció la intención de boicotear los productos. Se confirmó que las mujeres presentan actitudes más negativas que los hombres en cuanto a los estereotipos retratados, en términos de ofensividad. Sin embargo, no hubo diferencia significativa entre el género femenino y masculino en lo que se refiere al impacto de la ofensividad sobre la imagen de la empresa o la intención de boicot.

Palabras clave: Propaganda. Papeles Femeninos Estereotipados. Imagen de la Empresa. Intención de Compra. Contexto Brasileño.

\section{Introdução}

A mensagem de venda comunicada pela propaganda precisa ser rápida e de fácil processamento (WINDELS, 2016). Muitas vezes, isso resulta no retrato estereotipado de homens e mulheres: enquanto a figura masculina é comumente associada a locais de trabalho e automóveis; a feminina costuma anunciar itens de uso doméstico (MATTHES; PRIELER; ADAM, 2016).

Com as transformações socioculturais ocorridas nas últimas décadas, a exemplo da maior inserção das mulheres no mercado de trabalho, críticas a respeito do retrato feminino na propaganda passaram a ser levantadas (GRAU; ZOTOS, 2016).

A partir de 1970, diversos pesquisadores interessaram-se pelo tema, que ainda permanece um importante tópico de estudo (HUHMANN; LIMBU, 2016, GRAU; ZOTOS, 2016), diante da persistência do emprego dessas representações em anúncios veiculados nos mais diversos países (MATTHES; PRIELER; ADAM, 2016).

A maior parte da literatura ainda consiste em análises de conteúdo, estudos que se baseiam em esquemas de codificação das imagens para identificar a presença de papéis estereotipados na propaganda e descrevê-los (KYROUSI; PANIGYRAKIS; PANOPOULOS, 2016, BAXTER; KULCZYNSKI; ILICIC, 2016). O volume de pesquisas 
acerca dos efeitos dessas propagandas sobre os consumidores é bem mais reduzido, sendo que um número ainda menor de estudos examinou as atitudes dos homens sobre o tema (THEODORIDIS et al., 2013). Portanto, levanta-se como problema de pesquisa: Quais as atitudes de consumidores brasileiros adultos sobre os papéis femininos retratados na propaganda em geral e suas possíveis consequências para a imagem do anunciante e a intenção de compra dos produtos anunciados?

Aliado à pergunta e com base na escala de Lundstrom e Sciglimpaglia (1977), temse como objetivo geral analisar o efeito das atitudes a papéis femininos na propaganda em geral sobre a intenção de compra (ou boicote) dos consumidores e a possível interferência da imagem da empresa nessa relação.

\section{Papéis de gênero na propaganda brasileira}

A partir da "segunda onda" do movimento feminista, entende-se que "sexo" representa as características físicas que distinguem fêmea e macho (BRISTOR; FISCHER, 1993), enquanto que "gênero" se refere a traços, atitudes, crenças e tendências comportamentais enraizadas acerca de duas ramificações: a masculina e a feminina (CARVALHO, 2009).

Os estereótipos de gênero refletem essas crenças e podem ser desmembrados em traços psicológicos, características físicas, papéis comportamentais e profissões (KNOLL; EISEND; STEINHAGEN, 2011, KYROUSI; PANIGYRAKIS; PANOPOULOS, 2016, SHINODA, 2017). Destaca-se aqui os papéis comportamentais ou de gênero, cujo reforço pelas representações midiáticas pode gerar consequências limitadoras para as mulheres (KNOLL; EISEND; STEINHAGEN, 2011).

Courtney e Lockeretz (1971), em seu artigo seminal, concluem pela existência de quatro dimensões do papel feminino na propaganda, quais sejam: o lugar de uma mulher é em casa; as mulheres não tomam decisões importantes; são dependentes de homens; e são retratadas como objetos sexuais.

Na propaganda televisiva brasileira, Matthes, Prieler e Adam (2016) descobriram que: a proporção de figuras masculinas foi ligeiramente superior à de personagens femininas; retratos masculinos eram associados a carros e itens tecnológicos; é mais provável o retrato de mulheres em ambientes domésticos e de homens em locais de trabalho (MATTHES; PRIELER; ADAM, 2016).

Já Shinoda (2017) analisou anúncios de revistas brasileiras de 1995, 2005 e 2015 e afirma ter havido uma ampliação dos papéis representados no decorrer do tempo. Em 1995, papéis decorativos representavam mais da metade dos retratos, ao passo que em 2015 nota-se um aumento de papéis não tradicionais.

Para fins de ilustração, apresenta-se um anúncio da Editora Minuano avaliado pelo Conselho Nacional de Autorregulamentação Publicitária (CONAR), em atenção a dezenas de reclamações de consumidores reportando sexismo (Figura 1). 
Figura 1 - Estereótipo “dona de casa”

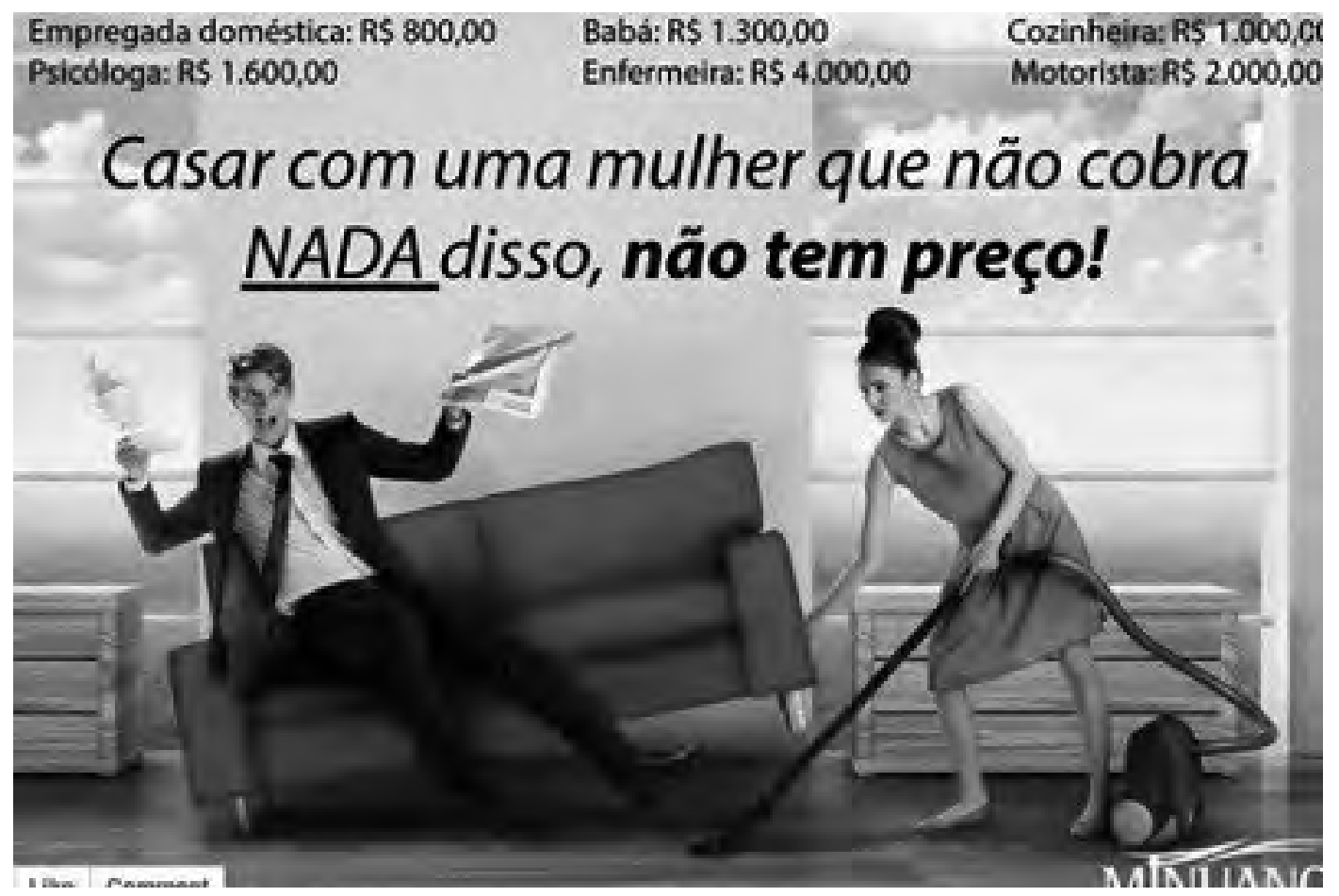

Fonte: O Globo (2016).

O anúncio passava a ideia de que "o homem casado com uma mulher que encara as funções de empregada doméstica, psicóloga, babá, enfermeira, cozinheira e motorista, se dá bem e acaba ‘economizando’ o que gastaria com essas profissionais” (O GLOBO, 2016, p. 1).

Em síntese, conquanto alguns estudos indiquem o aumento de representações igualitárias, em geral, papéis estereotipados ainda estão presentes na propaganda nacional.

\section{Atitudes dos consumidores a papéis femininos na propaganda em geral}

Sob a perspectiva do anunciante, uma propaganda é efetiva quando gera atitudes positivas em relação aos produtos ofertados e convence os consumidores a comprá-los (MORRISON; SHAFFER, 2003).

As atitudes a papéis de gênero consistem nas crenças de uma pessoa sobre direitos e responsabilidades de homens e mulheres (PALAN, 2001). No contexto da propaganda, tais atitudes podem envolver a percepção de inadequação dos papéis representados, ofensa e propagação de sexismo (FORD; LATOUR, 1996; FORD; LATOUR; HONEYCUTT JR., 1997; LATOUR; HENTHORNE; WILLIAMS, 1998; HUHMANN; LIMBU, 2016). Logo, 
a presente pesquisa lida com atitudes inerentemente críticas ou negativas ou "nível de criticismo”, nas palavras de Ford, Latour e Honeycutt Jr. (1997).

A adequação ou precisão (adequacy ou accuracy) diz respeito ao retrato de mulheres e homens como realmente são ou desempenhando as atividades que costumam realizar no dia a dia (FORD; LATOUR, 1996; FORD; LATOUR; HONEYCUTT JR., 1997; LATOUR; HENTHORNE; WILLIAMS, 1998).

As atitudes dos consumidores aos papéis de gênero na propaganda em geral são denominadas de atitudes gerais ou a priori, pois se referem à propaganda como um todo, ou seja, a um nível agregado (ORTH; HOLANCOVA, 2004). O estudo das atitudes em geral é relevante devido à sua influência sobre as respostas a anúncios específicos (ORTH; HOLANCOVA, 2004; THEODORIDIS et al., 2013).

Trabalhos que examinaram os efeitos de propagandas com estereótipos proporcionaram resultados mistos (EISEND; PLAGEMANN; SOLLWEDEL, 2014). Retratos estereotipados podem ser vantajosos ou prejudiciais, a depender de fatores como a cultura e as orientações individuais de gênero (BAXTER; KULCZYNSKI; ILICIC, 2016).

Pesquisas que consideraram respondentes dos dois genêros apontam que as mulheres são mais conscientes sobre os estereótipos na propaganda e apresentam atitudes mais negativas do que os homens (PLAGEMANN; SOLLWEDEL, 2014). Estes são mais propensos a crer que as propagandas retratam os gêneros em suas atividades diárias com precisão (HUHMANN; LIMBU, 2016). Também já se constatou que a ofensa provocada por retratos degradantes pode causar reações negativas às empresas e marcas anunciantes, a exemplo de atos de repúdio e boicote (CHRISTY, 2006).

\section{Imagem da Empresa e Intenção de Compra ou Boicote}

A construção de uma boa imagem institucional está relacionada à decisão de compra de potenciais consumidores e à criação de vantagem competitiva (GOMES; SAPIRO, 2003). Intenção de compra é a probabilidade de um indivíduo vir a experimentar ou adquirir a marca ou o produto anunciado num futuro próximo e não necessariamente implica no ato efetivo de compra (FERRELL; HARTLINE, 2010). Nesse contexto, imagem de marca é um construto holístico formado a partir de uma interpretação Gestalt de todas as associações relacionadas ao nome, termo, sinal ou símbolo utilizados com a intenção de identificar os bens e serviços de um determinado vendedor (KOTLER, 1997; FAIRCLOTH; CAPELLA; ALFORD, 2001). Em oposição à compra tem-se o boicote, ou seja, o ato de deixar de adquirir um produto ou serviço como forma de repúdio (CRUZ; PIRES JR.; ROSS, 2013).

Jones e Reid (2010) apontam que propagandas que se utilizam de estereótipos considerados degradantes afetam negativamente a intenção de compra das mulheres, como também de uma parcela substancial dos homens. Outros artigos indicam que mulheres 
relataram menor intenção de compra quando os anúncios continham retratos estereotipados (HUHMANN; LIMBU, 2016).

\section{Metodologia}

A partir do referencial teórico adotado, foram elaboradas as seguintes hipóteses:

$\mathbf{H}_{1}$ : No Brasil, as mulheres expressam atitudes mais negativas aos papéis femininos na propaganda do que os homens.

$\mathbf{H}_{2}$ : As atitudes a papéis femininos na propaganda têm efeito positivo sobre a intenção de boicote.

$\mathbf{H}_{3}$ : As atitudes a papéis femininos na propaganda têm efeito indireto positivo sobre a intenção de boicote através da imagem negativa da empresa (mediadora).

$\mathbf{H}_{4}$ : O gênero do respondente modera o efeito indireto (imagem da empresa) das atitudes a papéis femininos na propaganda sobre a intenção de boicote. Especificamente, para mulheres (vs. homens), o efeito indireto positivo será maior (vs. menor).

Com base em tais hipóteses construiu-se o modelo da pesquisa (Figura 2).

Figura 2 - Modelo de pesquisa

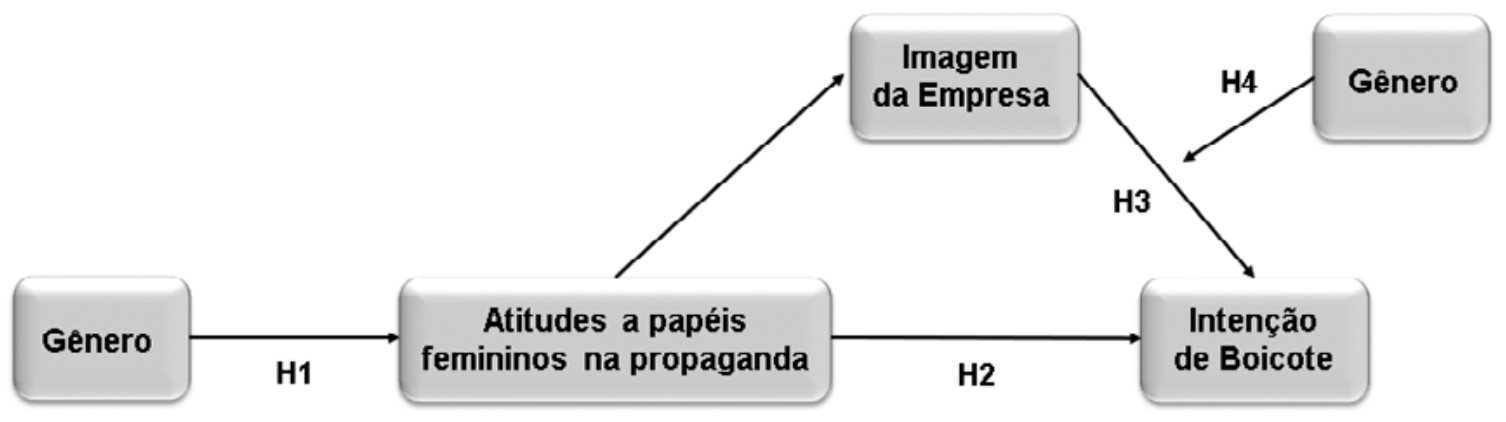

Fonte: Adaptado de Ford e Latour (1996), Ford, Latour e Honeycutt Jr. (1997) e Latour, Henthorne e Williams (1998).

Considera-se que as atitudes aos papéis femininos na propaganda em geral impactam a intenção de boicote de forma direta ou indireta, neste último caso, por meio da imagem negativa da empresa enquanto variável mediadora. Entende-se que o gênero dos(as) respondentes modera o efeito indireto esperado.

Trata-se de uma pesquisa descritiva, de abordagem quantitativa e horizonte temporal transversal, realizada por meio de survey ou levantamento. A população-alvo consiste em homens e mulheres adultos e residentes no Brasil. Deyoung e Crane (1992) afirmam que uma amostra mais representativa a nível nacional poderia oferecer melhores insights acerca do tema. Assim, se buscou alcançar homens e mulheres de todo o País, com idades e níveis de renda relativamente distintos. 
Como "lista ou conjunto de instruções para identificar a população-alvo" (MALHOTRA, 2012, p. 303), optou-se pela seleção de e-mails de docentes atuantes em universidades federais brasileiras nos cursos de Administração e Psicologia. Pensou-se que assim haveria uma maior taxa de retorno dos questionários, em razão de os respondentes estarem inseridos no meio acadêmico e em áreas relacionadas ao tema.

Logo, a amostra foi não probabilística e por conveniência (HAIR JR. et al., 2016). A partir dos parâmetros de Hair Jr. et al. (2016) e Kline (2011) para viabilizar uma análise fatorial confirmatória, calculou-se o tamanho da amostra em torno de 136 ou 204 elementos.

Os questionários foram estruturados pela escala de Lundstrom e Sciglimpaglia (1977), uma das mais utilizadas pela literatura para avaliar a recepção de estereótipos de gênero (NAVARRO-BELTRÁ; LLAGUNO, 2012). Realizou-se a tradução reversa da escala, com o auxílio de uma professora brasileira bilíngue e de um professor nativo dos Estados Unidos e fluente em português (Quadro 1).

Quadro 1 - Escala traduzida

\begin{tabular}{|l|l|}
\hline Atitudes a retratos de papéis de gênero na propaganda & Itens \\
\hline As propagandas que eu vejo mostram as mulheres como elas realmente são. & AT_1 \\
\hline $\begin{array}{l}\text { As propagandas sugerem que mulheres são fundamentalmente dependentes } \\
\text { dos homens. }\end{array}$ & AT_2 \\
\hline As propagandas que eu vejo mostram os homens como eles realmente são. & AT_3 \\
\hline As propagandas tratam as mulheres principalmente como “objetos sexuais”. & AT_4 \\
\hline $\begin{array}{l}\text { As propagandas que eu vejo retratam de forma fiel as mulheres na maioria } \\
\text { de suas atividades diárias. }\end{array}$ & AT_5 \\
\hline As propagandas sugerem que as mulheres tomam decisões importantes. & AT_6 \\
\hline $\begin{array}{l}\text { As propagandas que eu vejo retratam de forma fiel os homens na maioria de } \\
\text { suas atividades diárias. }\end{array}$ & AT_7 \\
\hline As propagandas sugerem que as mulheres não fazem coisas importantes. & AT_8 \\
\hline As propagandas sugerem que o lugar de uma mulher é em casa. & AT_9 \\
\hline $\begin{array}{l}\text { Eu estou mais sensível à maneira como as mulheres são retratadas na } \\
\text { propaganda do que eu costumava ser. }\end{array}$ & AT_10 \\
\hline $\begin{array}{l}\text { Eu considero ofensiva a forma como as mulheres costumam ser retratadas } \\
\text { na propaganda. }\end{array}$ & AT_11 \\
\hline $\begin{array}{l}\text { Em geral, eu acredito que o retrato das mulheres na propaganda está } \\
\text { mudando para melhor. }\end{array}$ & AT_12 \\
\hline
\end{tabular}




\begin{tabular}{|l|l|}
\hline Imagem negativa da empresa & Itens \\
\hline $\begin{array}{l}\text { Eu acredito que empresas que retratam a mulher de forma ofensiva em suas } \\
\text { propagandas são mais propensas a discriminar mulheres e outras minorias } \\
\text { em promoções e avanços profissionais, quando comparadas a outras } \\
\text { empresas no mesmo ramo ou mesma indústria. }\end{array}$ & IE_1 \\
\hline $\begin{array}{l}\text { Eu acredito que a maneira como a mulher é retratada nas propagandas } \\
\text { apenas reflete a atitude geral dessa empresa com relação ao lugar das } \\
\text { mulheres na sociedade. }\end{array}$ & IE_2 \\
\hline Intenção de boicote & Itens \\
\hline $\begin{array}{l}\text { Se um novo produto for introduzido por meio de propagandas que eu ache } \\
\text { ofensivas às mulheres, eu ainda posso vir a comprá-lo, caso me ofereça } \\
\text { benefícios que eu considere atrativos. }\end{array}$ & IB_1 \\
\hline $\begin{array}{l}\text { Se um novo produto ou serviço que eu já uso passar a adotar uma campanha } \\
\text { publicitária que eu considere ofensiva às mulheres, vou interromper a sua } \\
\text { utilização. }\end{array}$ & IB_2 \\
\hline $\begin{array}{l}\text { Mesmo que eu veja uma propaganda de um determinado produto e a } \\
\text { considere ofensiva às mulheres, eu continuaria a comprar outros produtos } \\
\text { que já venho utilizando da mesma empresa. }\end{array}$ & IB_3 \\
\hline
\end{tabular}

Fonte: Traduzido de Lundstrom e Sciglimpaglia (1977).

A escala é composta por 17 itens esparsos em três dimensões. Cada item é mensurado por uma escala tipo Likert de sete pontos (“discordo totalmente” até "concordo totalmente”). Um pré-teste do instrumento de coleta foi implementado para identificar a necessidade de modificações.

A coleta de dados via e-mail ocorreu entre os dias 13 de novembro e 29 de dezembro de 2017. Para cada destinatário, foi enviado um convite para participar do estudo por meio do link do formulário. Dos 2.124 questionários enviados, foram obtidas 537 respostas (taxa de retorno de aproximadamente 25\%).

Constatou-se a ocorrência de um missing value, ou seja, valor em branco ou ausente (GASKIN, 2017), em apenas um questionário, referente ao gênero. Haja vista que tal variável é fundamental para responder aos objetivos da pesquisa, o questionário foi excluído. Foram identificados outliers em 17 questionários, os quais também foram desconsiderados, de modo que se chegou uma amostra final de 519 sujeitos. Vale esclarecer que outliers, também denominados valores atípicos ou discrepantes, são respostas extremas a questionamentos específicos ou respostas extremas a todas as perguntas e que, portanto, devem ser interpretados no contexto do estudo (HAIR JR. et al., 2016).

Os cálculos de assimetria e curtose, mediante auxílio do IBM ${ }^{\circledR}$ SPSS Statistics ${ }^{\circledR}$ 20.0 para testar a normalidade, resultaram em valores dentro do limite de \pm 1,96 sugerido por Hair Jr. et al. (2016), indicando que a distribuição dos dados se aproximou da normal. 


\section{Perfil da Amostra}

Dentre os 519 sujeitos da amostra final, 280 (53,95\%) são do gênero feminino e 239 (46,05\%) do masculino. Quanto à faixa etária, a maior parte, isto é, 434 (83,62\%) pessoas, se encontra entre 31 e 60 anos. Com relação ao nível de renda, 247 (47,59\%) respondentes recebem de $\mathrm{R} \$ 9.370,00$ a $\mathrm{R} \$ 18.740,00$ por mês. Na sequência, estão aqueles em que a renda varia de $\mathrm{R} \$ 3.748,00$ a $\mathrm{R} \$ 9.370,00$, que correspondem a 200 (38,54\%) elementos. Houve predomínio de respondentes do Sudeste (27,17\%) e Nordeste (24,47\%). Em seguida., encontra-se o Sul (19,08\%) e o Centro-Oeste $(15,03 \%)$. Apenas 9,25\% da amostra afirmou pertencer ao Norte e 5,01\% não respondeu a esta questão.

\section{Análise Fatorial Exploratória (AFE)}

Uma AFE foi implementada para identificar construtos e eliminar a redundância (AAKER; KUMAR; DAY, 2001). Embora pesquisas anteriores (FORD; LATOUR, 1996; FORD; LATOUR; HONEYCUTT JR., 1997; LATOUR; HENTHORNE; WILLIAMS, 1998) já tenham realizado essa análise, optou-se por refazê-la, pois as estruturas fatoriais de tais estudos variaram quanto à composição de indicadores dos fatores. Aplicou-se o procedimento de componentes principais e a rotação varimax.

Após a exclusão dos itens "AT_10" e "AT_12" por não apresentarem cargas fatoriais significantes (maiores ou iguais a 0,5), obteve-se KMO de 0,684 e significância da esfericidade Bartlertt igual a 0,000. Todas as cargas fatoriais ficaram acima de 0,5 (HAIR JR. et al., 2016). Utilizou-se como parâmetro de aceitação dos Eigenvalues (autovalores) o valor superior a 1,0 (HAIR JR. et al., 2016), de modo foram obtidos os escores da Tabela 1.

Tabela 1 - Eigenvalues (Autovalores)

\begin{tabular}{|c|c|c|c|c|c|c|c|c|c|}
\hline \multicolumn{10}{|c|}{ Variância Total Explicada } \\
\hline \multicolumn{4}{|c|}{ Eigenvalues iniciais } & \multicolumn{3}{|c|}{$\begin{array}{l}\text { Soma da extração das cargas ao } \\
\text { quadrado }\end{array}$} & \multicolumn{3}{|c|}{$\begin{array}{l}\text { Soma das rotações das cargas } \\
\text { ao quadrado }\end{array}$} \\
\hline Fator & Total & $\begin{array}{l}\text { \% } \\
\text { Variância }\end{array}$ & $\begin{array}{l}\% \\
\text { Acumulado }\end{array}$ & Total & $\begin{array}{l}\text { \% } \\
\text { Variância }\end{array}$ & $\begin{array}{l}\% \\
\text { Acumulado }\end{array}$ & Total & $\begin{array}{l}\text { \% } \\
\text { Variância }\end{array}$ & $\begin{array}{l}\text { \% } \\
\text { Acumulado }\end{array}$ \\
\hline 1 & 4,14 & 27,57 & 27,57 & 4,14 & 27,57 & 27,57 & 3,17 & 21,15 & 21,15 \\
\hline 2 & 2,41 & 16,04 & 43,61 & 2,41 & 16,04 & 43,61 & 2,72 & 18,11 & 39,26 \\
\hline 3 & 2,10 & 14,00 & 57,61 & 2,10 & 14,00 & 57,61 & 2,39 & 15,95 & 55,20 \\
\hline 4 & 1,19 & 7,93 & 65,54 & 1,19 & 7,93 & 65,54 & 1,55 & 10,33 & 65,54 \\
\hline
\end{tabular}

Fonte: Dados da pesquisa (2017). 
Assim, uma solução aceitável resultou em quatro fatores, visto que a primeira dimensão da escala (“Atitudes a papéis femininos na propaganda”) se dividiu em dois fatores. Foi encontrada a mesma quantidade de variáveis latentes, bem como matrizes fatoriais similares às já verificadas pela literatura. Por tais razões, optou-se por utilizar, com pequenas adaptações, os mesmos rótulos empregados em estudos anteriores, quais sejam: “Ofensividade dos retratos de papéis femininos” (Fator 1); “Adequação dos retratos” (Fator 2); “Imagem negativa da empresa” (Fator 3) e "Intenção de boicote” (Fator 4).

Por fim, calculou-se o alfa de Cronbach de cada um dos fatores para verificar a precisão ou consistência interna das subescalas. Os valores de alfa foram todos superiores ao mínimo aceitável de 0,70 (HAIR JR. et al., 2016).

A Tabela 2 demonstra os resultados obtidos pela AFE.

Tabela 2 - Matriz fatorial e alfa de cronbach

\begin{tabular}{|c|c|c|c|}
\hline Construto & Item & Carga Fatorial & $\begin{array}{l}\text { Alfa de } \\
\text { Cronbach }\end{array}$ \\
\hline \multirow{6}{*}{1 - Ofensividade dos retratos de papéis femininos } & AT_8 & 0,814 & \multirow{6}{*}{0,806} \\
\hline & AT_9 & 0,748 & \\
\hline & AT_11 & 0,736 & \\
\hline & AT_4 & 0,667 & \\
\hline & AT_2 & 0,632 & \\
\hline & AT_6 & 0,532 & \\
\hline \multirow{4}{*}{2 - Adequação dos retratos } & AT_3 & 0,832 & \multirow{4}{*}{0,798} \\
\hline & AT_1 & 0,785 & \\
\hline & AT_7 & 0,761 & \\
\hline & AT_5 & 0,748 & \\
\hline \multirow{2}{*}{3 - Imagem negative da empresa } & CI_1 & 0,906 & \multirow{2}{*}{0,723} \\
\hline & CI_2 & 0,761 & \\
\hline \multirow{3}{*}{4 - Intenção de boicote } & BI_1 & 0,896 & \multirow{3}{*}{0,854} \\
\hline & BI_3 & 0,870 & \\
\hline & BI_2 & 0,838 & \\
\hline
\end{tabular}

Fonte: Dados da pesquisa (2017).

Para realizar a validação do modelo de mensuração por construto, foi implementada, em seguida, uma Análise Fatorial Confirmatória (AFC). 


\section{Análise Fatorial Confirmatória (AFC)}

Quando existem estudos anteriores, a AFC é indicada para confirmar, em uma nova amostra ou contexto, a estrutura fatorial já conhecida na literatura (BIDO; MANTOVANI; COHEN, 2016).

A escala de mensuração foi considerada reflexiva (CHIN, 1998) e a direção de “causalidade” partiu dos construtos para seus indicadores (HAIR JR. et al., 2016). Inicialmente, testou-se um modelo contendo os fatores 1 e 2 como variáveis latentes de primeira ordem que formavam uma de segunda ordem denominada "Atitudes a papéis femininos na propaganda". Contudo, tal modelo apresentou solução inadmissível pela presença de Heywood Cases (estimativas de parâmetros com valores ilógicos) (KLINE, 2011).

Logo, optou-se por um novo desenho do modelo sem a variável de segunda ordem. Nesta segunda tentativa, nenhum Heywood Case foi gerado. Foram aplicados alguns índices de modificação para melhorar o Model Fit (ajuste do modelo) e eliminou-se o item “AT_6” por apresentar baixa carga fatorial $(0,59)$ e ser considerado redundante (redação muito similar a “AT_8”), a fim de aprimorar ainda mais o ajuste e evitar problemas de validade.

As medidas de ajuste do modelo obtidas na segunda tentativa foram satisfatórias até para os parâmetros mais exigentes de Hu e Bentler (1999): CMIN/DF = 2,044; GFI = 0,952; $\mathrm{CFI}=0,970$; RMSEA = 0,051; e PCLOSE =0,421 .

Passou-se, então, à verificação da confiabilidade composta (CC) e validade do modelo. O primeiro cálculo de Variância Média Extraída (VME) resultou no valor de 0,486 (abaixo de 0,50) para “Adequação dos retratos”. Percebeu-se que o indicador “AT_7” apresentava carga fatorial baixa $(0,39)$, não sendo verdadeiramente representativo da variável latente. Assim, foi eliminado do modelo e, novamente, foram calculadas as medidas de validade e confiabilidade que, desta vez, foram todas adequadas (HAIR JR. et al., 2016).

Na Tabela 3, os números em bold são as raízes quadradas das VMEs e números fora da diagonal são os valores das correlações entre os construtos.

Tabela 3 - Validade e Confiabilidade do Modelo

\begin{tabular}{l|l|l|l|l|l|l}
\hline & CC & VME & $\begin{array}{l}\text { Imagem da } \\
\text { Empresa }\end{array}$ & Ofensividade & Adequação & $\begin{array}{l}\text { Intenção } \\
\text { de Boicote }\end{array}$ \\
\hline $\begin{array}{l}\text { Imagem da } \\
\text { Empresa }\end{array}$ & 0,794 & 0,669 & $\mathbf{0 , 8 1 8}$ & & & \\
\hline Ofensividade & 0,846 & 0,525 & 0,492 & $\mathbf{0 , 7 2 4}$ & & \\
\hline Adequação & 0,811 & 0,599 & 0,208 & 0,479 & $\mathbf{0 , 7 7 4}$ & \\
\hline
\end{tabular}

1 CMIN/DF corresponde ao valor do teste qui-quadrado (chi-square) divido pelo número de graus de liberdade (degrees of freedom); GFI (Goodness-of-fit Index ou índice de qualidade de ajuste); CFI (Comparative Fit Index ou índice de ajuste comparativo); RMSEA (Root Mean Square Approximation ou raiz da média dos quadrados dos erros de aproximação); SRMR (Standardized root mean square residual ou raiz quadrada média residual); PCLOSE ( $p$ of close fit). 


\begin{tabular}{l|l|l|l|l|l|l}
\hline & CC & VME & $\begin{array}{l}\text { Imagem da } \\
\text { Empresa }\end{array}$ & Ofensividade & Adequação & $\begin{array}{l}\text { Intenção } \\
\text { de Boicote }\end{array}$ \\
\hline $\begin{array}{l}\text { Intenção de } \\
\text { Boicote }\end{array}$ & 0,852 & 0,660 & 0,320 & 0,311 & 0,226 & $\mathbf{0 , 8 1 2}$ \\
\hline
\end{tabular}

Fonte: Dados da pesquisa (2017).

O ajuste do modelo sem o item “AT_7” foi verificado mais uma vez, apresentando índices significativos (HU; BENTLER, 1999), vide Tabela 4.

Tabela 4 - Ajuste do modelo de mensuração final

\begin{tabular}{l|l|l}
\hline Parâmetro & Referência & Observado \\
\hline CMIN/DF & $<3,0$ & 2,106 \\
\hline GFI & $>0,95$ & 0,954 \\
\hline CFI & $>0,95$ & 0,97 \\
\hline RMSEA & $<0,05$ bom; $0,50-0,10$ moderado & 0,053 \\
\hline PCLOSE & $>0,05$ & 0,351 \\
\hline Standardized RMR & $<0,90$ & 0,047 \\
\hline
\end{tabular}

Fonte: Dados da pesquisa (2017).

A estrutura final da escala contou com 13 itens e a solução fatorial de quatro construtos obtida na fase exploratória foi, até então, mantida (Figura 3). 
Figura 3 - Modelo de mensuração final

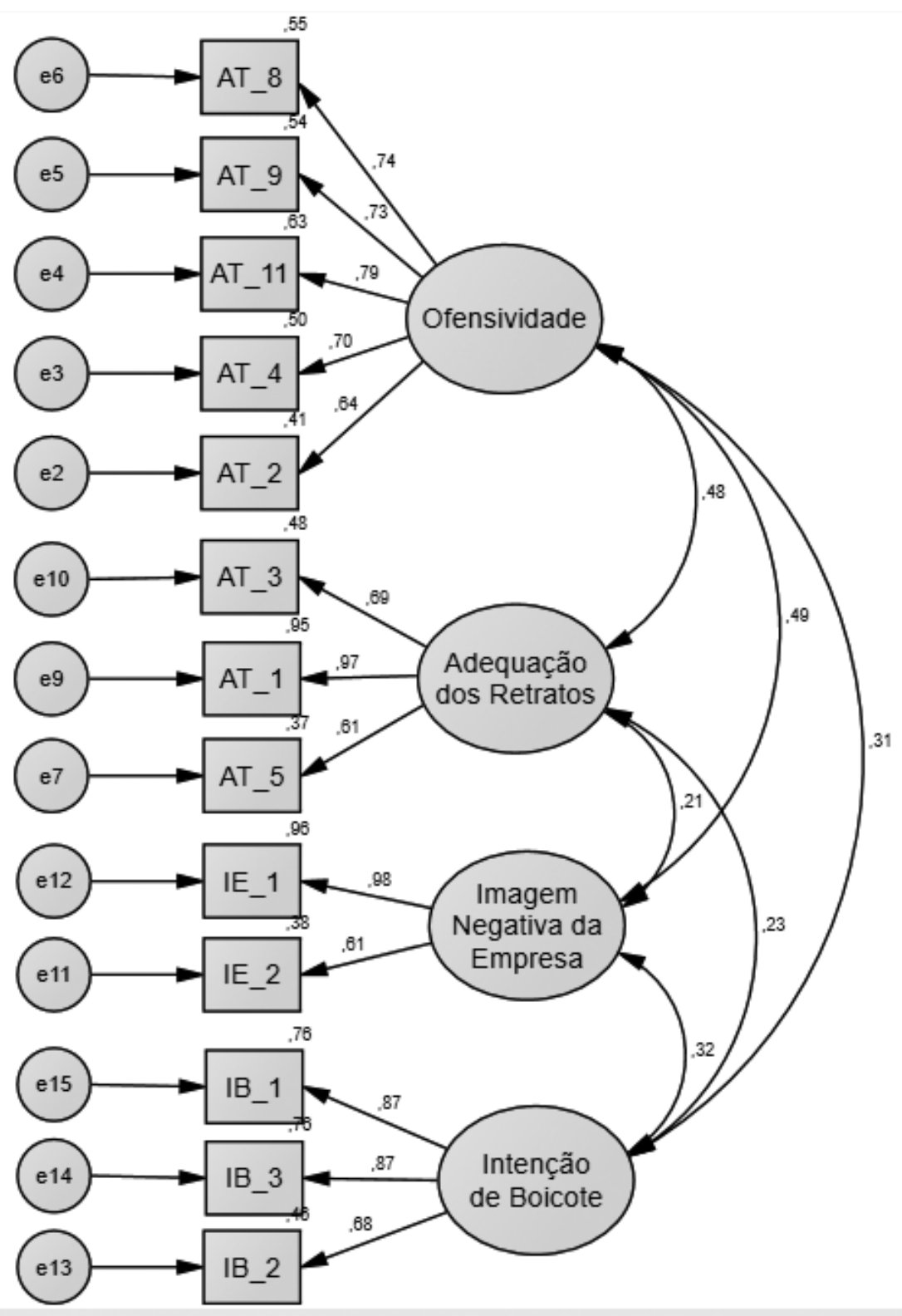

Fonte: Dados da pesquisa (2017). 
Haja vista que, para o teste das hipóteses, é necessária a realização de uma análise multigrupo (feminino e masculino), antes é preciso testar a invariância do modelo. O teste de invariância métrica nos moldes de Kline (2011) resultou em um valor não significativo de P $(0,442)$. Isso evidenciou que os grupos não foram diferentes ao nível do modelo, contudo, ainda poderiam apresentar distinções na análise de caminhos. Assim, existe equivalência no modelo de mensuração entre os grupos e as respostas entre homens e mulheres podem ser comparadas.

\section{Modelo Estrutural e Verificação das Hipóteses}

A fim de testar as hipóteses, utilizou-se o método de Structural Equation Modeling (SEM), o qual é indicado para analisar relações de dependência entre variáveis latentes (HAIR JR. et al., 2016). Optou-se pela SEM baseada em covariância (CB-SEM), por meio do IBM ${ }^{\circledR}$ SPSS ${ }^{\circledR}$ Amos v. 20.0, que envolve o critério da Máxima Verossimilhança, pois a distribuição dos dados se aproximou da normal.

No presente estudo, foi verificado se, conforme o gênero, o efeito das atitudes a papéis femininos na propaganda em geral (variável independente) sobre a intenção de boicote, por meio da imagem negativa da empresa, poderia ser reduzido, trazido a zero (valor de $\mathrm{P}$ não significativo) ou, até mesmo, adquirir sinal invertido (+/-). Logo, diante de uma variável independente contínua atuando juntamente com uma varíavel modedora dicotômica (gênero), Baron e Kenny (1986) sugerem um modelo multigrupo em SEM.

O fator "imagem negativa da empresa" foi tido como moderador, visto que se trata de uma terceira variável que intervém entre dois outros construtos relacionados (HAIR JR. et al., 2016). Portanto, tem-se uma mediação moderada. Iacobucci, Saldanha e Deng (2007) indicam que a melhor maneira de analisar mediação moderada é, justamente, por meio de SEM e que alguns softwares já possuem uma opção de sintaxe para esse tipo de cálculo por meio de modelos multigrupo. Neste trabalho, foi utilizada a ferramenta Multigroup Analysis disponível no IBM ${ }^{\circledR}$ SPSS ${ }^{\circledR}$ Amos v. 20.0.

Uma primeira análise dos pesos de regressão não padronizados demonstrou que o impacto da variável “Adequação dos retratos” não foi significativo $(p>0,01)$ para a predição de nenhum dos demais construtos, conforme evidenciaram os valores de C.R. (critical ratios) e de P (Tabela 5). Para que alcancem significância, os valores de C.R. devem ser superiores a 1,96 (BYRNE, 2010).

Tabela 5 - Pesos de regressão não padronizados (Adequação de retratos)

\begin{tabular}{l|l|l|l|l}
\hline Relação & Estimativa & S.E. & C.R. & P valor \\
\hline Adequação_retratos --> Imagem_empresa & $-0,051$ & 0,08 & $-0,641$ & 0,522 \\
\hline Adequação_retratos --> Intenção_boicote & 0,180 & 0,103 & 1,756 & 0,079 \\
\hline
\end{tabular}

Fonte: Dados da pesquisa (2017). 
Embora se tenha alcançado um bom ajuste do modelo de mensuração com o construto "Adequação dos retratos", optou-se por descartar esse fator para o teste do modelo estrutural (Figura 4), pois foi corroborada a conclusão de Ford e Latour (1996), Ford, Latour e Honeycutt (1997) e de Latour, Henthorne e Williams (1998) quanto a sua não significância.

Figura 4 - Modelo Estrutural Final

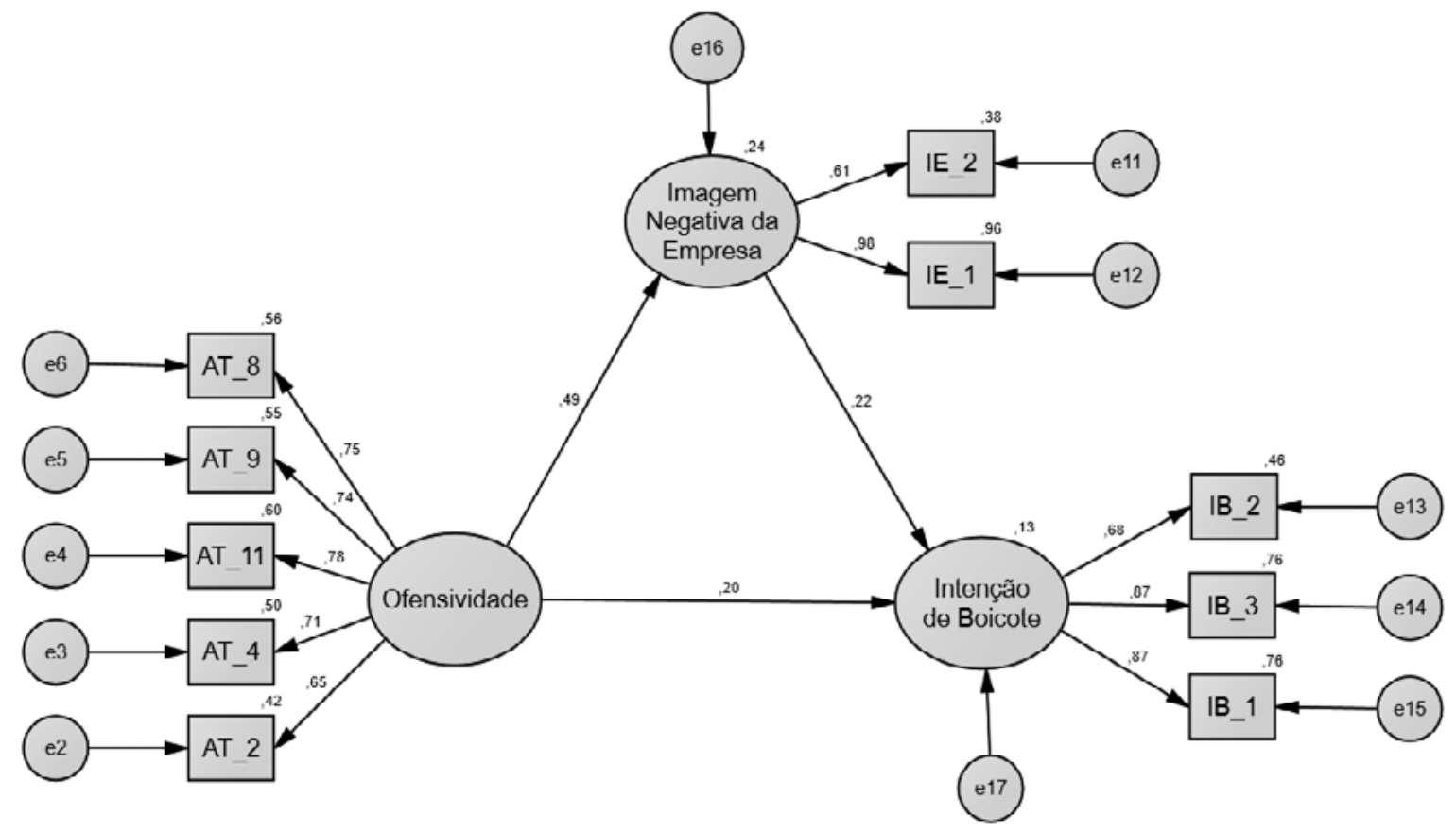

Fonte: Dados da pesquisa (2017).

O ajuste do modelo final sem a "Adequação de retratos” gerou valores adequados (HU; BENTLER, 1999), vide Tabela 6.

Tabela 6 - Ajuste do modelo estrutural final

\begin{tabular}{l|l|l}
\hline Parâmetro & Referência & Observado \\
\hline CMIN/DF & $<3,0$ & 1,715 \\
\hline GFI & $>0,95$ & 0,974 \\
\hline CFI & $>0,95$ & 0,986 \\
\hline RMSEA & $<0,05$ bom; 0,50 - 0,10 moderado & 0,042 \\
\hline PCLOSE & $>0,05$ & 0,731 \\
\hline Standardized RMR & $<0,90$ & 0,032 \\
\hline
\end{tabular}

Fonte: Dados da pesquisa (2017). 
Assim, restou assegurada a possibilidade de testar as hipóteses. Ante a desconsideração da “Adequação de retratos”, tem-se que as “Atitudes negativas em relação ao retrato de papéis femininos na propaganda” são refletidas apenas pelo grau de “Ofensividade dos retratos de papéis femininos" manifestado pelos respondentes.

A primeira hipótese foi testada no IBM ${ }^{\circledR}$ SPSS Statistics ${ }^{\circledR}$ por meio de um Teste $\mathrm{T}$ entre duas amostras independentes (gênero masculino e feminino) com relação ao construto “Ofensividade dos retratos de papéis femininos”. Para isso, criou-se uma variável dependente pelo método aditivo, abarcando todos os indicadores do fator (AT_2, AT_4, AT_8, AT_9, AT_11). O teste de Levene para equivalência de variâncias foi significativo ( $F=0,001$; Sig. = 0,971; $\mathrm{p}>0,05)$. Logo, foram considerados os valores de teste $\mathrm{T}$ assumindo variâncias iguais, que constam na Tabela 7. Infere-se, pelo valor de p <0,01 (sig. 0,000) que há diferença significativa no grau de ofensividade dado o gênero.

Tabela 7 - Teste $\mathrm{T}$ de amostras independentes para $\mathrm{H}_{1}$

\begin{tabular}{l|l|l|l|l|l|l}
\hline \multicolumn{2}{l|}{} & $\mathbf{t}$ & $\begin{array}{l}\text { Graus de } \\
\text { liberdade }\end{array}$ & Sig. & $\begin{array}{l}\text { Diferença } \\
\text { de média }\end{array}$ & $\begin{array}{l}\text { Diferença } \\
\text { de erro de } \\
\text { padrão }\end{array}$ \\
\hline \multirow{2}{*}{ Ofensividade } & $\begin{array}{l}\text { Assumindo } \\
\text { equivalência de } \\
\text { variâncias }\end{array}$ & 5,02 & 398 & 0 & 3,16776 & 0,63101 \\
\hline
\end{tabular}

Fonte: Dados da pesquisa (2017).

Os valores de média demonstram que o grupo feminino expressou um grau de ofensividade levemente maior que o masculino (Tabela 8).

Tabela 8 - Estatísticas de grupo

\begin{tabular}{l|l|l|l|l|l}
\hline & $\begin{array}{l}\text { Identidade de } \\
\text { Gênero }\end{array}$ & $\mathbf{N}$ & Média & Desvio Padrão & Média Erro Padrão \\
\hline \multirow{2}{*}{ Ofensividade } & Feminino & 217 & 23,8618 & 6,35371 & 0,43132 \\
\cline { 2 - 6 } & Masculino & 183 & 20,6940 & 6,20743 & 0,45887 \\
\hline
\end{tabular}

Fonte: Dados da pesquisa (2017).

Adequando tais escores à escala de 7 pontos utilizada (dividindo-se os valores de média por 7), tem-se que a média final do grupo feminino foi de 3,41 e a do masculino foi de 2,96. Assim, confirma-se $\mathrm{H}_{1}$ no que tange às atitudes que refletem ofensividade.

Em $\mathrm{H}_{2}$ e $\mathrm{H}_{3}$ analisa-se a significância das relações entre as variáveis latentes e em $\mathrm{H}_{4}$, o efeito moderador do gênero. Desse modo, aquelas hipóteses são verificadas no contexto do modelo total, enquanto que esta passa por uma análise multigrupos (feminino e masculino). 
A análise dos pesos de regressão do modelo global (Tabela 9) demonstrou efeitos significativos entre "Ofensividade" $\mathrm{e}$ "Imagem negativa da empresa" $(\beta=0,489 ; \mathrm{p}<0,001)$ e "Imagem negativa da empresa” e "Intenção de boicote” $(\beta=0,221 ; \mathrm{p}<0,001)$. A relação entre “Ofensividade” e “Intenção de boicote” $(\beta=0,203)$ também pode ser considerada significativa a um $\mathrm{p}<0,01$. Foram parcialmente suportadas, portanto, $\mathrm{H}_{2}$ e $\mathrm{H}_{3}$, de forma que a “Ofensividade dos retratos de papéis femininos” foi preditora direta da “Intenção de boicote”, bem como indireta, quando parcialmente mediada pela "Imagem negativa da empresa". Tem-se uma mediação parcial, diante da significância de todas as relações.

Tabela 9 - Parâmetros estimados no modelo total

\begin{tabular}{l|l|l|l|l|l}
\hline & $\begin{array}{l}\text { Peso } \\
\text { padronizado } \\
\text { de regressão }\end{array}$ & $\begin{array}{l}\text { Peso não } \\
\text { padronizado } \\
\text { de regressão }\end{array}$ & S.E. & C.R. & $\begin{array}{l}\text { P } \\
\text { valor }\end{array}$ \\
\hline Ofensividade --> Intenção_boicote & 0,203 & 0,236 & 0,08 & 2,962 & 0,003 \\
\hline Ofensividade --> Imagem_empresa & 0,489 & 0,488 & 0,086 & 5,66 & $* * *$ \\
\hline $\begin{array}{l}\text { Imagem_empresa --> Intenção_ } \\
\text { boicote }\end{array}$ & 0,221 & 0,257 & 0,075 & 3,448 & $* * *$ \\
\hline
\end{tabular}

Fonte: Dados da pesquisa (2017).

Para verificação de $\mathrm{H}_{4}$, ao se dividir a amostra nos dois grupos (217 feminino e 183 masculino), surgiram Heywood Cases no grupo feminino relacionados à "Imagem negativa da empresa” (variância de “e12” = - 0,398). Corrigiu-se a situação pelas sugestões de Dillon, Mulani e Kumar (1987) e, então, foi possível rodar o modelo contendo ambos os grupos, o qual alcançou bom ajuste (CMIN/DF =1,268; GFI = 0,962; CFI = 0,988; RMSEA = 0,026; PCLOSE = 0,997; SRMR = ,0422).

Por meio da ferramenta Multiple-Group Analysis foi realizado um teste de quiquadrado entre o modelo totalmente restrito e o modelo estimado livremente (GASKIN, 2017), cujo resultado ( $p=0,132)$ indica não haver diferença significativa entre os grupos feminino e masculino com relação ao modelo global (Tabela 10).

Tabela 10 - Teste qui-quadrado no modelo global

\begin{tabular}{l|l|l|l|l|l|l|l}
\hline \multirow{2}{*}{ Model } & \multirow{2}{*}{ DF } & \multirow{2}{*}{ CMIN } & \multirow{2}{*}{ P } & NFI & IFI & RFI & TLI \\
\cline { 5 - 8 } & & & & Delta-1 & Delta-2 & rho-1 & rho2 \\
\hline Structural weights & 10,000 & 15,005 & 0,132 & 0,009 & 0,010 & 0,002 & 0,002 \\
\hline
\end{tabular}

Fonte: Dados da pesquisa (2017).

Por preciosismo, partiu-se para uma análise de caminhos. As relações de interesse foram, uma a uma, forçadas a serem equivalentes entre os grupos (enquanto as demais eram 
estimadas livremente) e, então, verificou-se as distinções entre eles (GASKIN, 2017). Os valores dos testes qui-quadrado podem ser conferidos na Tabela 11. Confirmou-se a ausência de diferença entre os grupos em todas as relações e conclui-se por refutar $\mathrm{H}_{4}$.

Tabela 11 - Teste qui-quadrado com relações restritas uma a uma

\begin{tabular}{|c|c|c|c|c|c|c|c|}
\hline & \multirow{2}{*}{ DF } & \multirow{2}{*}{ CMIN } & $\mathbf{P}$ & NFI & IFI & RFI & TLI \\
\hline & & & valor & Delta-1 & Delta-2 & rho-1 & rho2 \\
\hline Ofensividade --> Intenção_boicote & 1 & 0,246 & 0,62 & 0 & 0 & $-0,001$ & $-0,001$ \\
\hline Ofensividade --> Imagem_empresa & 1 & 0,719 & 0,396 & 0 & 0,000 & 0 & 0 \\
\hline $\begin{array}{l}\text { Imagem_empresa --> Intenção_ } \\
\text { boicote }\end{array}$ & 1 & 0,217 & 0,642 & 0 & 0 & $-0,001$ & $-0,001$ \\
\hline
\end{tabular}

Fonte: Dados da pesquisa (2017).

Esclarece-se que $\mathrm{H}_{1}, \mathrm{H}_{2}$ e $\mathrm{H}_{3}$ foram apenas parcialmente aceitas, pois o fator “Adequação dos retratos” teve de ser descartado e, portanto, as atitudes a papéis femininos na propaganda foram avaliadas somente em termos do nível de ofensividade percebido. Ademais, a mediação prevista em $\mathrm{H}_{3}$ foi, na prática, apenas parcial e não total.

Por meio do teste de $\mathrm{H}_{1}$, constatou-se que, no Brasil, o grupo feminino apresentou atitudes mais negativas ao retrato de papéis estereotipados, aqui mensuradas especificamente por um maior grau de ofensividade. Logo, restaram reforçadas as conclusões de pesquisas prévias (LUNDSTROM; SCIGLIMPAGLIA, 1977; LATOUR; HENTHORNE; WILLIAMS, 1998; FORD; LATOUR; MIDLETON, 1999; THEODORIDIS et al., 2013; EISEND; PLAGEMANN; SOLLWEDEL, 2014).

$\mathrm{O}$ teste de $\mathrm{H}_{2}$ revelou que as atitudes a papéis femininos na propaganda em geral possuem uma relação direta e significativa com a intenção de boicote, em termos de ofensividade. Em complemento, verificou-se que essa relação pode sofrer interferência parcial da imagem negativa da empresa $\left(\mathrm{H}_{3}\right)$.

Ao contrário do previsto, os resultados do teste de $\mathrm{H}_{4}$ indicaram não haver diferença significativa entre homens e mulheres nas relações de impacto da ofensividade sobre a intenção de boicote, por meio da imagem negativa da empresa. Nesse ponto, não foram corroborados os resultados de Ford e Latour (1996) e Latour, Henthorne e Williams (1998).

O Quadro 2 sintetiza os resultados dos testes das hipóteses.

Quadro 2 - Resultados dos testes de hipóteses

\begin{tabular}{|l|l|}
\hline Hipóteses & Resultado \\
\hline $\begin{array}{l}\mathbf{H}_{\mathbf{1}}: \text { No Brasil, as mulheres expressam atitudes mais negativas aos papéis } \\
\text { femininos na propaganda do que os homens }\end{array}$ & $\begin{array}{l}\text { Parcialmente } \\
\text { aceita }\end{array}$ \\
\hline
\end{tabular}




\begin{tabular}{|c|c|}
\hline Hipóteses & Resultado \\
\hline $\begin{array}{l}\mathbf{H}_{2}: \text { As atitudes a papéis femininos na propaganda têm efeito positivo sobre a } \\
\text { intenção de boicote }\end{array}$ & $\begin{array}{l}\text { Parcialmente } \\
\text { aceita }\end{array}$ \\
\hline $\begin{array}{l}\mathbf{H}_{3}: \text { As atitudes a papéis femininos na propaganda têm efeito indireto } \\
\text { positivo sobre a intenção de boicote através da imagem negativa da empresa } \\
\text { (mediadora) }\end{array}$ & $\begin{array}{l}\text { Parcialmente } \\
\text { aceita }\end{array}$ \\
\hline $\begin{array}{l}\mathbf{H}_{4} \text { : O gênero do respondente modera o efeito indireto (imagem da empresa) } \\
\text { das atitudes a papéis femininos na propaganda sobre a intenção de boicote. } \\
\text { Especificamente para mulheres (vs. homens), o efeito indireto positivo será } \\
\text { maior (vs. menor). }\end{array}$ & Refutada \\
\hline
\end{tabular}

Fonte: Elaborado pelos autores (2018).

De modo geral, constatou-se que há, dentre uma amostra de consumidores brasileiros, preocupação significativa a respeito da temática, a qual pode gerar repercussões relevantes para o sucesso das comunicações no País.

\section{Conclusões}

Este estudo corrobora a estrutura fatorial encontrada por pesquisas prévias. Também confirma as relações entre as variáveis latentes, ou seja, a ofensividade evocada pelo retrato de papéis femininos estereotipados na propaganda, em geral, influenciou diretamente a intenção de boicotar os produtos e serviços anunciados, bem como indiretamente, por meio da imagem negativa da empresa, cuja mediação parcial se confirmou.

Ademais, este trabalho soma à corrente de pesquisa referente aos efeitos de retratos estereotipados na propaganda sobre os consumidores e moderniza o instrumento de Lundstrom e Sciglimpaglia (1977) ao aplicá-lo em um cenário atual e culturalmente diferente. Ao utilizar uma amostra de respondentes das cinco regiões do Brasil, esta pesquisa atende às sugestões de Deyoung e Crane (1992), Orth e Holancova (2004) e Tsichla e Zotos (2016), pois expande o estudo do tema e dá mais um passo em direção a uma possível universalização dos resultados. Em especial, este estudo representa uma primeira tentativa de suprir a carência de trabalhos sobre gênero e propaganda no contexto sul-americano (URIBE et al., 2008). Representa, também, um avanço para pesquisas sobre boicote no País, ainda pouco realizadas (CRUZ; PIRES JR.; ROSS, 2013).

As descobertas deste trabalho também auxiliam anunciantes a desenvolverem estratégias de comunicação mais efetivas, diante da ciência de que papéis estereotipados em comerciais podem intensificar a percepção negativa da imagem corporativa no que tange ao tratamento despendido a suas colaboradoras mulheres e quanto à visão da empresa acerca do lugar da figura feminina na sociedade. 
Além disso, os resultados indicam uma reação comportamental negativa de mulheres e homens brasileiros a essas empresas, no sentido de evitarem a compra ou descontinuarem o uso de produtos ou serviços.

Ainda, vale comentar as possíveis repercussões regulatórias dos achados deste estudo, que evidencia a relevância dos retratos de gênero para a ofensividade da propaganda brasileira. Diante das recentes decisões do CONAR quanto a denúncias de sexismo, reforçase a noção de que os anunciantes precisam investigar as atitudes a papéis de gênero de seus targets (HUHMANN; LIMBU, 2016).

Como limitações, este trabalho investigou atitudes a um nível agregado. Logo, os resultados não são destinados a um segmento do mercado ou categoria de produto da propaganda em particular. Diante da incipiência dos estudos sobre o tema no Brasil, considerou-se suficientemente interessante esta análise geral num primeiro momento, porém, pesquisas futuras podem examinar a questão de forma mais singularizada.

Cabe ponderar que as percepções dos respondentes podem variar muito, a depender de características como hábitos de consumo, faixa etária, cor ou raça, renda e níveis de acesso às peças publicitárias veiculadas nos mais diversos meios de comunicação - haja vista que alguns desses meios podem estar mais abertos a representações progressistas de gênero do que outros. Inclusive, os dados de renda obtidos podem indicar um recorte de classe na pesquisa. Tais características, embora não explícitas neste estudo, podem influenciar os resultados obtidos. Somado à utilização de uma amostra não probabilística, isso impede a realização de generalizações para toda a população.

A escala utilizada também pode ser aperfeiçoada no que tange à "Imagem negativa da empresa”, cuja composição por apenas dois itens pode ter sido a causa para o surgimento de Heywood Cases.

Por fim, é preciso atentar para o fato de que esta é uma pesquisa de caráter transversal. Novos estudos devem realizar testes longitudinais, a fim de verificar mudanças de atitude ao longo do tempo.

\section{Referências}

AAKER, D. A.; KUMAR, V.; DAY, G. S. Pesquisa de Marketing. São Paulo: Atlas, 2001.

BAXTER, S. M.; KULCZYNSKI, A.; ILICIC, J. Ads aimed at dads: exploring consumers' reactions towards advertising that conforms and challenges traditional gender role ideologies. International Journal of Advertising, [S.l.], v. 35, n. 6, p. 970-982, 2016.

BRISTOR, J. M.; FISCHER, E. Feminist thought: Implications for consumer research. Journal of consumer research, [S.1.], v. 19, n. 4, p. 518-536, 1993.

BYRNE, B. M. Structural equation modeling with AMOS: Basic concepts, applications and programming. [S.l.]: Routledge, 2010.

CARVALHO, M. G. de et al. (org.). Construindo a igualdade na diversidade: gênero e sexualidade na escola. Curitiba: Editora da UTFPR, p. 21-32, 2009. 
CHIN, W. W. The partial least squares approach to structural equation modeling. Modern methods for business research, v. 295, n. 2, p. 295-336, 1998.

CHRISTY, T. P. Females' perceptions of offensive advertising: The importance of values, expectations, and control. Journal of current issues \& research in advertising, v. 28, n. 2, p. 15-32, 2006.

CRUZ, B. de P. A.; ROSS, S. D.; PIRES JR., R. J. M. Diferença de gênero na Percepção de Culpa no boicote de consumidores. Revista Brasileira de Gestão de Negócios, [S.l.], v. 15, n. 49, p. 504-523, 2013.

DEYOUNG, S.; CRANE, F. G. Females’ Attitudes Toward the Portrayal of Women in Advertising: A Canadian Study. International Journal of Advertising, [S.l.], v. 11, n. 3, p. 249-255, jan. 1992.

DILLON, W. R.; KUMAR, A.; MULANI, N. Offending estimates in covariance structure analysis: Comments on the causes of and solutions to Heywood cases. Psychological Bulletin, v. 101, n. 1, p. 126, 1987.

EISEND, M.; PLAGEMANN, J.; SOLLWEDEL, J. Gender roles and humor in advertising: The occurrence of stereotyping in humorous and nonhumorous advertising and its consequences for advertising effectiveness. Journal of advertising, [S.1.], v. 43, n. 3, p. 256-273, 2014.

FAIRCLOTH, J. B.; CAPELLA, L. M.; ALFORD, B. L. The effect of brand attitude and brand image on brand equity. Journal of marketing theory and practice, v. 9, n. 3, p. 61-75, 2001.

FERRELL, O. C.; HARTLiNE, M. D. Estratégia de Marketing. São Paulo: Cengage Learning, 2010.

FORD, J. B.; LATOUR, M. S. Contemporary female perspectives of female role portrayals in advertising. Journal of Current Issues \& Research in Advertising, [S.l.], v. 18, n. 1, p. 81-95, 1996.

FORD, J. B.; LATOUR, M. S.; HONEYCUTT JR., E. D. An examination of the cross-cultural female response to offensive sex role portrayals in advertising: A research note. International Marketing Review, [S.l.], v. 14, n. 6, p. 409-423, 1997.

GASKIN, J. Structural Equation Modeling: Multi-group. 2017. Disponível em: http://statwiki. kolobkreations.com/index.php?title=Structural_Equation_Modeling\#Multi-group. Acesso em: 3 jan. 2018.

GRAU, S. L.; ZOTOS, Y. C. Gender stereotypes in advertising: a review of current research. International Journal of Advertising, [S.l.], v. 35, n. 5, p. 761-770, 2016.

HAIR JR., J. F. et al. A primer on partial least squares structural equation modeling (PLS-SEM). Sage Publications, 2016.

HUHMANN, B. A.; LIMBU, Y. B. Influence of gender stereotypes on advertising offensiveness and attitude toward advertising in general. International Journal of Advertising, [S.l.], v. 35, n. 5, p. 846863, 21 mar. 2016.

IACOBUCCI, D.; SALDANHA, N.; DENG, X. A meditation on mediation: Evidence that structural equations models perform better than regressions. Journal of Consumer Psychology, v. 17, n. 2, p. 139-153, 2007.

JONES, S. C.; REID, A. The use of female sexuality in Australian alcohol advertising: public policy implications of young adults' reactions to stereotypes. Journal of Public Affairs, [S.l.], v. 10, n. 1-2, p. 1935, 26 out. 2009.

KLINE, R. B. Principles and practice of structural equation modeling. 3. ed. New York: The Guilford Press, 2011.

KNOLL, S.; EISEND, M.; STEINHAGEN, J. Gender roles in advertising: Measuring and comparing gender stereotyping on public and private TV channels in Germany. International Journal of Advertising, [S.l.], v. 30, n. 5, p. 867-888, 2011. 
KOTLER, P. Marketing management: Analysis, planning, implementation and control. 1997. Disponível em: https://alphamedia.technology/show/marketing-management-analysis-planning-implement-1.html. Acesso em: 13 maio 2017.

KYROUSI, A. G.; PANIGYRAKIS, G. G.; PANOPOULOS, A. P. Attitudes toward ads portraying women in decorative roles and female competition: an evolutionary psychology perspective. International Journal of Advertising, [S.l.], v. 35, n. 5, p. 771-798, 2016.

LATOUR, M. S.; HENTHORNE, T. L.; WILLIAMS, A. J. Is industrial advertising still sexist: It's in the eye of the beholder. Industrial Marketing Management, [S.l.], v. 27, n. 3, p. 247-255, 1998.

LUNDSTROM, W. J.; SCIGLIMPAGLIA, D. Sex role portrayals in advertising. Journal of Marketing, [S.l.], p. 72-79, 1977.

MALHOTRA, N. K. Pesquisa de marketing: uma orientação aplicada. Bookman Editora, 2012.

MATTHES, J.; PRIELER, M.; ADAM, K. Gender-role portrayals in television advertising across the globe. Sex roles, [S.l.], v. 75, n. 7-8, p. 314-327, 2016.

MORRISON, M. M.; SHAFFER, D. R. Gender-role congruence and self-referencing as determinants of advertising effectiveness. Sex Roles, [S.l.], v. 49, n. 5, p. 265-275, 2003.

NAVARRO-BELTRÁ, M.; LLAGUNO, M. M. A systematic review of gender and advertising studies. Catalan Journal of Communication \& Cultural Studies, [S.l.], v. 2, n. 4, p. 171-183, 2012.

O GLOBO. Conar atende a denúncias e susta anúncio considerado machista. 2016. Disponível em: https:// blogs.oglobo.globo.com/gente-boa/post/conar-atende-denuncias-e-susta-anuncio-considerado-machista. html. Acesso em: 10 maio 2017.

ORTH, U. R.; HOLANCOVA, D. Men's and women's responses to sex role portrayals in advertisements. International Journal of Research in Marketing, [S.l.], v. 21, n. 1, p. 77-88, 2004.

PALAN, K. M. Gender identity in consumer behavior research: A literature review and research agenda. Academy of Marketing Science Review, [S.l.], 2001.

SHINODA, L. M. Padrões de representação do estereótipo do gênero feminino na propaganda brasileira. 2017. 161 f. Dissertação (Mestrado) - Curso de Administração de Empresas, Fundação Getúlio Vargas, São Paulo, 2017.

THEODORIDIS, P. K. et al. Male and female attitudes towards stereotypical advertisements: a paired country investigation. Corporate Communications: An International Journal, [S.l.], v. 18, n. 1, p. 135160, 25 jan. 2013.

TSICHLA, E.; ZOTOS, Y. Gender portrayals revisited: searching for explicit and implicit stereotypes in Cypriot magazine advertisements. International Journal of Advertising, [S.l.], v. 35, n. 6, p. 9831007, 2016.

URIBE, R. et al. Estereotipos de género en la publicidad: un análisis de contenido de las revistas chilenas. Academia. Revista Latinoamericana de Administración, [S.1.], n. 41, 2008.

WINDELS, K. Stereotypical or just typical: how do US practitioners view the role and function of gender stereotypes in advertisements? International Journal of Advertising, v. 35, n. 5, p. 864-887, 2016. 


\section{Fernanda Almeida Marcon}

Mestra pelo Programa de Pós-Graduação em Administração, na linha de pesquisa de Marketing e Estratégia, pela Universidade Federal de Santa Catarina (PPGA/UFSC-2018). Pós-graduada em Direito Constitucional pela Universidade Candido Mendes (UCAM-2017) e em Jurisdição Federal pela Escola Superior da Magistratura Federal de Santa Catarina (ESMAFESC-2016). Graduada em Direito pela Universidade Federal de Santa Catarina (UFSC-2015) e em Administração Empresarial pela Universidade do Estado de Santa Catarina (UDESC-2014). Realizou o curso Entrepreneurship, Creativity \& Innovation, na Itália (Fondazione CUOA2012). Atuou como Tutora de Educação à Distância no Curso de Graduação em Administração e no Programa de Capacitação “O Saber para conquistar um lugar” (UFSC-2016/2017). Membra do grupo de pesquisa registrado na UFSC e no CNPq, denominado: Núcleo de Inteligência Competitiva Organizacional em Marketing e Logística (NICO). E-mail: fernanda@ almeidamarcon.com.

\section{Rudimar Antunes da Rocha}

Possui pós-doutorado em Administração pela Escola de Economia e Gestão da Universidade do Minho (EEG-U.Minho (2013-2014) - Braga/Portugal - Bolsa Estágio Sênior Capes. Doutor em Engenharia de Produção pela Universidade Federal de Santa Catarina (PPGEP-UFSC-2000 - Bolsa Capes). Mestre em Administração pela Universidade Federal de Santa Catarina (CPGA-UFSC-1992 - Bolsa Capes) e Bacharel em Administração pela Universidade Federal de Santa Maria (UFSM-1982). Professor titular do Curso de Ciências da Administração (CAD/ UFSC) e do Programa de Pós-Graduação em Administração (PPGA/UFSC). Atuou como professor do Programa de Pós-Graduação em Administração Universitária (PPGAU/UFSC). Autor de diversos artigos publicados em revistas científicas e anais de eventos nacionais e internacionais. Líder do grupo de pesquisa registrado na UFSC e no CNPq, denominado: Núcleo de Inteligência Competitiva Organizacional em Marketing e Logística (NICO). E-mail: rrudimar@gmail.com.

\section{Ana Maria Simões Ribeiro}

Doutoranda pelo Programa de Pós-Graduação em Administração, na linha de pesquisa de Produção e Desenvolvimento, pela Universidade Federal de Santa Catarina (PPGA/UFSC). Mestra pelo Programa de Pós-Graduação em Administração, na linha de pesquisa de Marketing e Estratégia, pela Universidade Federal de Santa Catarina (PPGA/UFSC). Graduanda em Administração Empresarial pela Universidade do Estado de Santa Catarina (UDESC). Graduada em Comunicação Social - Publicidade e Propaganda, pela Universidade Federal de Minas Gerais (UFMG-2015) e em Comunicação Social - Jornalismo e Relações Públicas, pela 
Universidade Federal de Minas Gerais (UFMG-2013). Tutora de Educação à Distância no Curso de Graduação em Administração, na Universidade Federal de Santa Catarina (UFSC). Membra do grupo de pesquisa registrado na UFSC e no CNPq, denominado: Núcleo de Inteligência Competitiva Organizacional em Marketing e Logística (NICO). E-mail: anasimoesribeiro@ gmail.com.

Recebido em: 15.02.2019 Aprovado em: 03.05.2021 